\title{
Effect of Rotation and Revolution on Performance of Blade-Free Planetary Mixer
}

\author{
Takayuki Yamagata, Nobuyuki Fujisawa \\ Faculty of Engineering, Niigata University, Niigata, Japan \\ Email: yamagata@eng.niigata-u.ac.jp
}

How to cite this paper: Yamagata, T. and Fujisawa, N. (2019) Effect of Rotation and Revolution on Performance of Blade-Free Planetary Mixer. Journal of Flow Control, Measurement \& Visualization, 7, 1-10. https://doi.org/10.4236/jfcmv.2019.71001

Received: August 11, 2018

Accepted: October 11, 2018

Published: December 14, 2018

Copyright $\odot 2019$ by authors and Scientific Research Publishing Inc. This work is licensed under the Creative Commons Attribution International License (CC BY 4.0).

http://creativecommons.org/licenses/by/4.0/

(c) (i) Open Access

\begin{abstract}
In this study, flow structures and mixing performance in a blade-free planetary mixer, which combines rotation and revolution motions inside a cylindrical vessel, are numerically investigated. Flow fields in the mixer vessel are simulated in a single rotating reference frame with various revolution speeds and a fixed rotation speed. The mixing process is investigated by a Lagrangian particle tracking method and the mixing performance is evaluated based on particle concentration. The results of the numerical simulations show that a vortical flow with an axis inclined with respect to the rotation axis of the vessel is generated by the combined influence of the rotation and revolution motions. The flow structure and vortical flow intensity vary as a function of the precession rate, which is the ratio of the revolution speed to rotation speed. The mixing performance of the blade-free planetary mixer is found to be maximum at aspecific precession rate.
\end{abstract}

\section{Keywords}

Blade-Free Planetary Mixer, Numerical Simulation, Particle Tracking, Flow Visualization, Mixing

\section{Introduction}

Liquid blending and solid-liquid mixing are important operations in the production processes of various industries such as chemical, pharmaceutical, and food. A typical method for the blending and mixing is agitation of a fluid in a vessel with impellers. Various techniques, such as visualization of mixing process [1] and velocity measurement by particle image velocimetry [2] [3] [4], have been used to clarify the mixing mechanism and improve mixing performance. Theoretical consideration [5] and numerical simulations of mixing performance [6]-[13] were also performed using computational fluid dynamics 
(CFD), which can be applied to prediction in practical problems. Although agitation is a typical mixing technique, there is a demand for blending and mixing without impellers and agitators for mixing liquids with higher viscosities.

A blade-free planetary mixer is a new technology for mixing liquids without impellers and agitators. This type of mixer can mix highly viscous fluids with less contamination as the flow in the vessel is induced by precession that combines rotation and revolution of the vessel. The fluid mechanics in a precessing cylinder, which is the basic principle of the blade-free planetary mixer, has been studied in connection with the instability of the fluid motion [14]. However, the design methodology of this type of mixer has not been thoroughly studied in literature, which may be due to the complexity of the flow field of the blade-free planetary mixer even in a laminar flow regime. Only a paper in literature is a numerical simulation of flow field in the blade-free planetary mixer of laminar flow [15]. It reported that spiral vortical flows were generated in the vessel, and their sizes, shapes, and rotation axes were influenced by the precession rate, which is the ratio of the revolution speed to the rotation speed. Nevertheless, the influence of the precession rate on laminar mixing performance has not been clarified, because it is difficult to evaluate the mixing performance only from the flow field information.

Two approaches to evaluate mixing performance with CFD have been reported in the literature: Eulerian-Lagrangian method [6] [7] [8] [9] [10] and Eulerian-Eulerian method [11] [12] [13]. The Eulerian-Lagrangian method was employed in this study, because this method is desirable for advection-dominated problems, and it can provide the trajectory of individual particles in the mixing process. An example of the Eulerian-Lagrangian method is the mixing performance analysis in a precessing sphere [10]. It was found that the flow mixing was enhanced under weak precession in the turbulent flow regime.

The objective of this study is to investigate the laminar mixing performance by numerical simulation in a blade-free planetary mixer, which is related to flow structures induced by the combined influence of rotation and revolution. The influence of the precession rate on the flow structure and mixing performance was also investigated.

\section{Numerical Methods}

\subsection{Flow Field}

Flow structures in a blade-freeplanetary mixer vessel were investigated by three-dimensional numerical simulations [16]. The simulations were carried out with the SRFPimpleFoam solver in OpenFOAM 4.1. The solver used in this study was an unsteady-state solver for incompressible Newtonian fluid flows including a rotating reference frame in the computational domain. However, the target flow field was the steady state laminar flow of blade-free planetary mixer. Fluid motion in a rotating frame is expressed by Navier-Stokes equations with the Coriolis and centrifugal forces acting as external forces, as well as continuity 
equation. The governing equations are written as follows:

$$
\begin{gathered}
\frac{\partial \boldsymbol{u}}{\partial t}+\boldsymbol{u} \cdot \nabla \boldsymbol{u}=-\frac{1}{\rho} \nabla p+v \nabla^{2} \boldsymbol{u}-2 \boldsymbol{\Omega} \times \boldsymbol{u}-\boldsymbol{\Omega} \times \boldsymbol{\Omega} \times \boldsymbol{r} \\
\nabla \cdot \boldsymbol{u}=0
\end{gathered}
$$

where $t, \boldsymbol{u}, p, \rho$, and $v$ denote time, fluid velocity, pressure, fluid density, and viscosity, respectively. Note that the fluid velocity and position vectors are defined as the relative velocity and position vectors in the rotating frame. The third term on the right-hand side of Equation (1) is the Coriolis force, where $\Omega$ is the revolution speed of the rotating frame. The fourth term is the centrifugal force, where $\boldsymbol{r}$ represents the position vector from the revolution axis.

Figure 1 shows the computational configuration of a blade-free planetary mixer. The mixing vessel has cylindrical geometry with an inner diameter of $d=$ $76 \mathrm{~mm}$ and a height of $h=77 \mathrm{~mm}$. The rotation axis of the vessel was inclined 45 degrees with respect to the revolution axis. The revolution radius was $R_{r e v}=$ $83 \mathrm{~mm}$ at the bottom center of the vessel. The configuration of the mixer was determined based on an actual machine designed for the purpose of experimental investigation. The rotation speed of the vessel was set to $n_{\text {rot }}=60 \mathrm{rpm}$, and the fluid was silicone oil with kinematic viscosity of $v=1.5 \times 10^{-5} \mathrm{~m}^{2} / \mathrm{s}$ to keep the flow regime laminar in the range of the computational conditions. Therefore, the Reynolds number was $\operatorname{Re}\left(=1 / 4 \cdot \omega_{\text {rot }} \cdot d^{2} / v\right)=605$, where $\omega_{\text {rot }}$ is the angular velocity. Note that the present Reynolds number is smaller than the critical Reynolds number of $1 \times 10^{4}$ in a stirred cylindrical vessel with weak precession [14]. Simulations were performed with revolution speeds of $n_{\text {rev }}=0,15,30,45$, and $60 \mathrm{rpm}$, and the precession rates in the simulation were $\Gamma\left(=n_{\mathrm{rev}} / n_{\mathrm{rot}}\right)=0$, $0.25,0.5,0.75$, and 1 . The rotation and revolution directions were opposite.

The computational domain was in the cylindrical vessel which was filled with the fluid. At the wall boundaries of the vessel, the rotation speed was applied to define the rotational motion of the vessel. The governing equations were discretized using the finite volume method with the quadratic upstream interpolation for convective kinematics (QUICK) scheme for the convective term and with the central differencing scheme for the other terms. The pressure implicit with splitting of

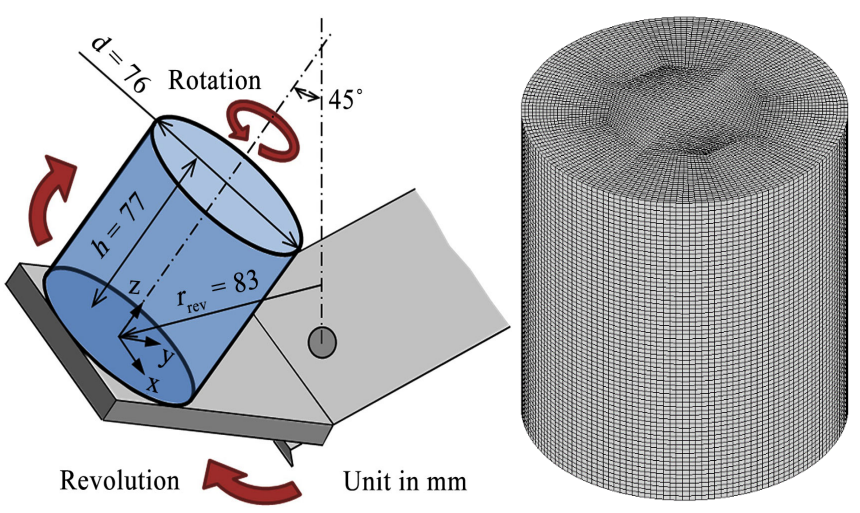

Figure 1. Computational configuration and grid of a planetary centrifugal mixer. 
operator (PISO) algorithm was used to solve the governing equations. Independence of the grid convergence was evaluated using fine, medium, and coarse grids with $6.5 \times 10^{5}, 3.8 \times 10^{5}$, and $4.8 \times 10^{4}$ cells, respectively. Root-mean-square errors for the velocity fields based on the fine grid were $0.1 \%$ and $1.8 \%$ for the medium grid and the coarse grid, respectively. Consequently, the medium grid shown in Figure 1 was used for numerical simulations in this study.

\subsection{Mixing Performance}

In this study, the mixing performance of the blade-free planetary mixer was evaluated by the Eulerian-Lagrangian method. The degree of mixing can be estimated from the number density distribution of particles by tracking a sufficient number of the particles in the vessel. Particle trajectories were simulated with the Lagrangian particle tracking method as passive particles after flow in the vessel reached the steady state. In this study, for experimental validation, it was assumed that solid particles gravitated to the bottom before mixing and were transported throughout the vessel by mixing. The particles were spherical with $10 \mu \mathrm{m}$ diameter, and the particle mass density was equal to the mass density of the fluid in order to evaluate the mixing performance of the flow field itself. Collisions between particles were ignored.

The mixing performance was evaluated by the mixing index, which was calculated based on the particle number density in the vessel [17]. The entire vessel was divided into $N$ sub-domains. The relative particle number density $\rho_{i}$ was defined as the ratio of the particle number density in the th sub-domain to the maximum particle number density in all sub-domains. The standard deviation of the number density $\sigma$ over all sub-domains is defined as follows:

$$
\sigma=\sqrt{\frac{1}{N} \sum_{i=1}^{N}\left(\rho_{i}-\rho_{m}\right)^{2}}
$$

where, $\rho_{m}$ represents the average particle number density in the entire vessel. The mixing index $I_{m}$ is given by the following equation:

$$
I_{m}=1-2 \sigma
$$

In this study, the number of sub-domains was $N=6000$ and the number of supplied particles was 6000 per second.

\section{Results and Discussion}

\subsection{Flow Structure}

Velocity fields in a blade-free planetary mixer vessel were computed to understand the mixing mechanism. Figure 2 shows three-dimensional flow structures and cross-sectional velocity fields in the mixer vessel for a rotation speed of $n_{r o t}$ $=60 \mathrm{rpm}$ and precession rates $\Gamma\left(=n_{\text {rev }} / n_{\text {rot }}\right)=0$ (a), 0.25 (b), 0.5 (c), and 1 (d). The results for $\Gamma=0.75$ are omitted because they are similar to the results for $\Gamma=$ 1. Streamlines (left) are shown to analyze the three-dimensional flow structures, and velocity magnitude contours of the in-plane velocity components are shown 

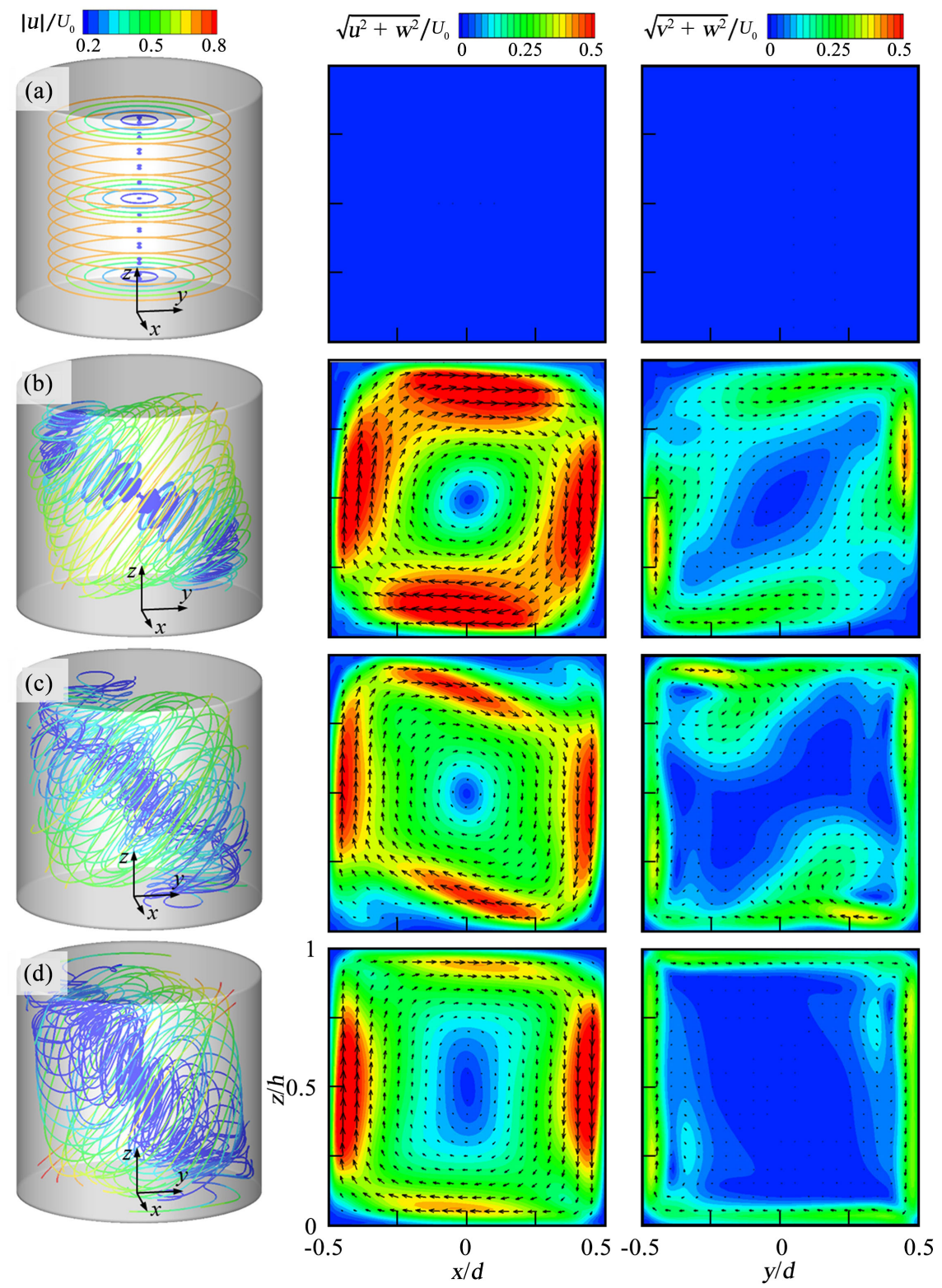

Figure 2. Flow structures and cross-sectional velocity fields: (a) $n_{\text {rev }}=0 \mathrm{rpm}(\Gamma=0)$, (b) $n_{\mathrm{rev}}=15 \mathrm{rpm}(\Gamma=0.25),(\mathrm{c}) n_{\mathrm{rev}}=30 \mathrm{rpm}(\Gamma=0.5)$, and (d) $n_{\mathrm{rev}}=60 \mathrm{rpm}(\Gamma=1)$. Each column shows the streamlines (left), velocity contours in the $x$ - $Z$ plane (middle), and in the $y$ - $z$ plane (right).

in the $x-z$ plane (middle) and in the $y-z$ plane (right). The velocity component was normalized by the wall velocity $U_{0}\left(=\frac{1}{2} d \omega_{\text {rot }}\right)$. At this Reynolds number, a steady flow was observed after a certain transient state. The results from panel (a) show circular streamlines parallel to the bottom and no in-plane velocity component due to the two-dimensional rotating flow. In the precession cases, the streamlines show the generation of vortical flows with axes inclined with respect to the spin axis of the vessel. The velocity fields for the lower precession rate of $\Gamma=0.25$ (b) show strong rotational flow in the vertical cross-section, but 
the flow structure was nearly two-dimensional. The results for $\Gamma=0.5$ (c) show a spiral axis of the rotating flow and a complex vortical flow structure. Non-axisymmetric rotating flow was observed in the $x-Z$ plane, and twin corner vortices occurred near the upper left and lower right corners in the $y$ - $z$ plane. As the precession rate increases further, the flow structure for $\Gamma=1$ (d) became more complex. However, the low-speed region extended around the vortical flow axis. These characteristics of the vortical flow structure with the spiral and inclined axis in the blade-free planetary mixer are similar to the results from others presented in the literature [15]. The occurrence of the rotating flows in the vertical cross-section of the cylindrical vessel is caused by the inclination of the axis of the rotating flow, which is caused by the Coriolis force due to revolution. It is noted that the rotating flow does not occur in the cylindrical vessel without revolution. With increasing the precession rate, the flow around the axis of the rotating flow is highly magnified due to the instability of the rotating flow [14]. Therefore, the complex flow pattern due to the Coriolis force is generated in the vessel and results in the enhancement of flow mixing in the blade-free planetary mixer.

\subsection{Mixing Performance}

The mixing performance was investigated by tracking tracer particles in the vessel and evaluating the particle number density. Figure 3 shows the temporal evolution of particle mixing in the vertical $(x-z)$ plane. The particle positions were calculated based on the velocity field at different precession rates of (a) $\Gamma=$ 0 , (b) 0.25 , (c) 0.5 , and (d) 1 . Particles were supplied near the bottom surface. The degree of mixing converged to a steady state in any cases by $t=200 \mathrm{~s}$ after mixing began. In the case of only rotation (a), the particles remained at the bottom of the vessel due to the two-dimensional rotational flow. In the case of $\Gamma=$ 0.25 (b), some particles moved to the upper region along the vessel walls by the rotating flow induced through the combined effect of rotation and revolution. However, the particles continued to move in the outer area along the vessel walls. This result indicates that although the rotating flow axis was inclined with respect to the vessel spin axis, the flow in the vessel was a two-dimensional rotating flow. As the revolution speed increased, the particles were dispersed throughout the vessel and particle mixing was maximized for $\Gamma=0.5$ (c). The relatively high concentration of particles was observed in the lower part of the vessel due to the supply of particles from the bottom surface. As the revolution speed increased further, unmixed areas were observed for $\Gamma=1$ (d) due to the low-speed rotating flow region in the $y$ - $z$ plane (Figure 2).

To quantify the mixing performance, the mixing index was evaluated from the particle number density at different precession rates. Figure 4 summarizes the mixing index in the steady state as the precession rate varies. As shown in the figure, the precession rate for $\Gamma=0.5$ shows the highest mixing index value. The mixing index at $\Gamma=0.25$ was comparatively lower, though strong rotating flow 
occurred in the vertical plane. At higher precession rates, the mixing index gradually decreased due to the occurrence of the unmixed region, since the low-speed region extended around the rotating flow axis. These results indicate that the moderate precession enhances the mixing performance by the magnified rotating flow, but too strong precession prevents the formation of the large-scale vortical flow. Therefore, an optimum precession rate, which is around $\Gamma=0.5$, is found to enhance the mixing performance of a blade-free planetary mixer. It should be mentioned that the optimum precession rate in a precessing sphere operating in a turbulent flow [10] was reported at $\Gamma=0.1$. Therefore, the optimum precession rates may be affected by the influence of container shapes, precession angles, Reynolds numbers and the laminar or turbulent flow. Therefore, further investigation is needed to clarify the influence of these parameters on the optimum precession rate.

$t=10 \mathrm{~s}$
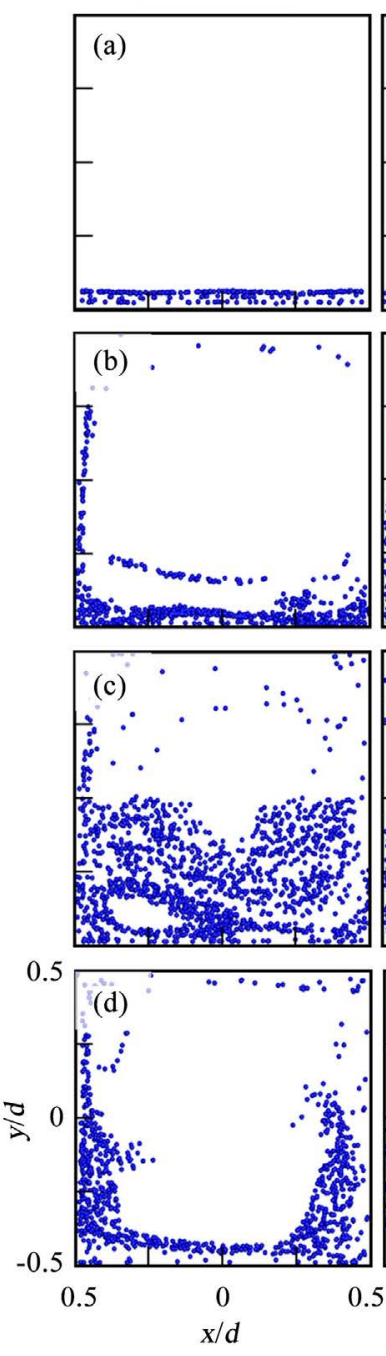

$t=50 \mathrm{~s}$
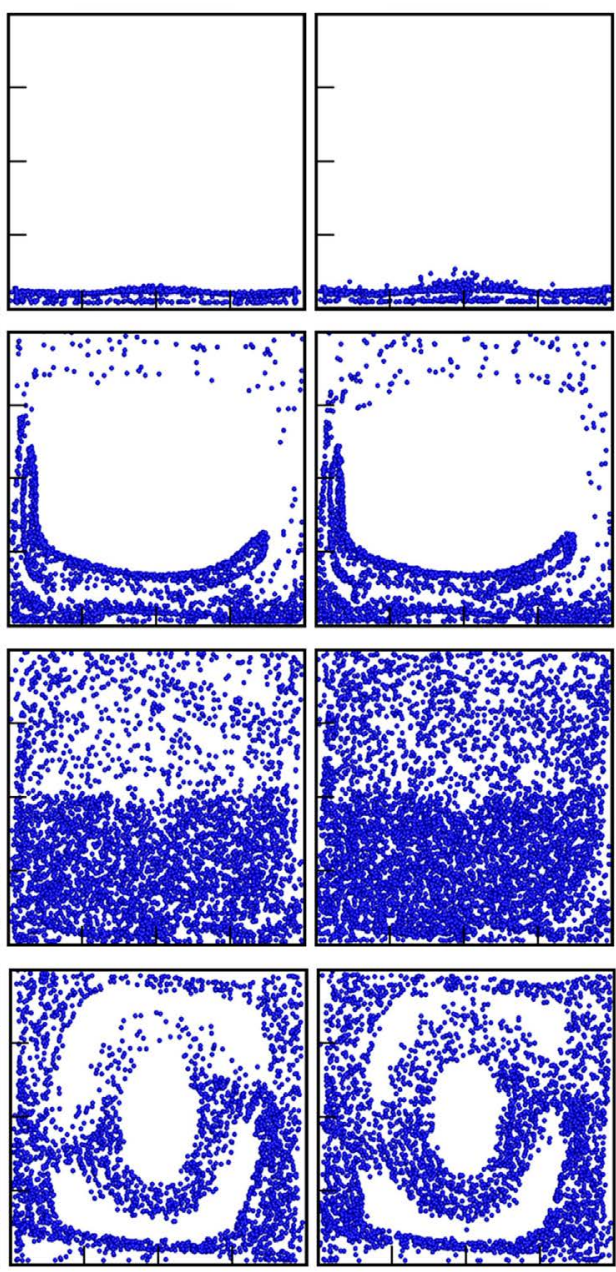

Figure 3. Temporal evolution of mixing of tracer particles from the bottom of the vessel: (a) $n_{\text {rev }}=0 \mathrm{rpm}(\Gamma=0)$, (b) $n_{\text {rev }}=15 \mathrm{rpm}(\Gamma=0.25)$, (c) $n_{\text {rev }}=30 \mathrm{rpm}(\Gamma=0.5)$, and (d) $n_{\text {rev }}=60 \mathrm{rpm}(\Gamma=1)$. 


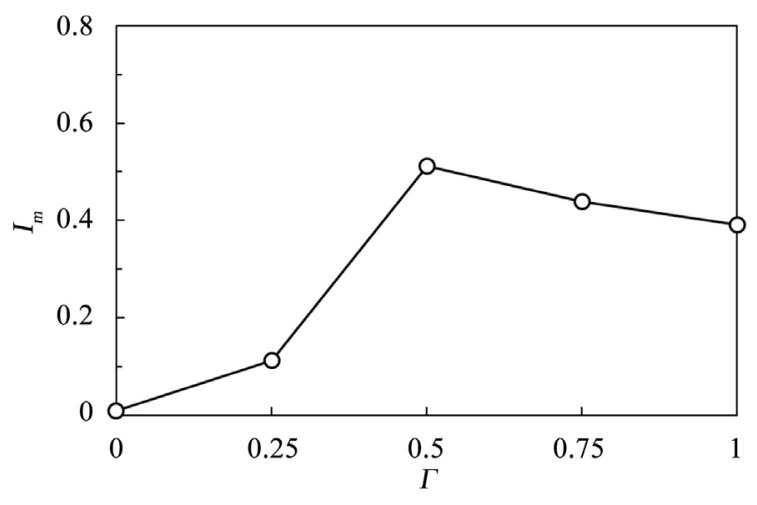

Figure 4. Mixing index versus precession rate.

\section{Conclusion}

The flow structures and the mixing performance of a blade-free planetary mixer were investigated by numerical simulations. The flow fields in the mixer vessel were determined by solving the Navier-Stokes equation considering the influence of the Coriolis and centrifugal forces in a rotating reference frame. The mixing process was numerically visualized by a particle tracking method, and the mixing index was evaluated from the concentration of particles. In the numerical results, a vortical structure with an axis inclined with respect to the rotation axis was induced by the combined influence of rotation and revolution at lower precession rates. This vortical flow generated a rotating flow in the vertical cross-section of the vessel. As the precession rate increases, vortical flows showed complex three-dimensional flow structures with a spiral rotation axis. It is found from the present numerical simulation that the laminar mixing performance of the blade-free planetary mixer becomes maximum around the precession rate 0.5 , which is contributed by the occurrence of rotating flow with an inclination angle to the spin axis and the generation of highly magnified flow around the axis caused by the precession. However, too strong precession prevents the formation of the large-scale vortical flow.

\section{Acknowledgements}

The authors express thanks to Mitsuboshi Co. Ltd. for the help and suggestions during the course of this study.

\section{Conflicts of Interest}

The authors declare no conflicts of interest regarding the publication of this paper.

\section{References}

[1] Wang, S., Wu, J. and Bong, E.Y. (2013) Reduced IMRs in a Mixing Tank via Agitation Improvement. Chemical Engineering Research and Design, 91, 1009-1017. https://doi.org/10.1016/j.cherd.2013.01.009

[2] Aubin, J., Le Sauze, N., Bertrand, J., Fletcher, D.F. and Xuereb, C. (2004) PIV Mea- 
surements of Flow in an Aerated Tank Stirred by a Down- and an Up-Pumping Axial Flow Impeller. Experimental Thermal and Fluid Science, 28, 447-456. https://doi.org/10.1016/j.expthermflusci.2001.12.001

[3] Zhu, H., Nienow, A.W., Bujalski, W. and Simmons, M.J.H. (2009) Mixing Studies in a Model Aerated Bioreactor Equipped with and Up- or a Down-Pumping "Elephant Ear" Agitator: Power, Hold-Up and Aerated Flow Field Measurement. Chemical Engineering Research and Design, 87, 307-317.

https://doi.org/10.1016/j.cherd.2008.08.013

[4] Guida, A., Nienow, A.W. and Barigou, M. (2010) The Effect of the Azimuthal Position of the Measurement Plane on the Flow Parameters Determined by PIV within a Stirred Vessel. Chemical Engineering Science, 65, 2454-2463.

https://doi.org/10.1016/j.ces.2009.12.012

[5] Nienow, A. (1997) On Impeller Circulation and Mixing Effectiveness in the Turbulent Flow Regime. Chemical Engineering Science, 52, 2557-2565.

https://doi.org/10.1016/S0009-2509(97)00072-9

[6] Aubin, J., Fletcher, D.F. and Xuereb, C. (2005) Design of Micromixers Using CFD Modelin. Chemical Engineering Science, 60, 2503-2516.

https://doi.org/10.1016/j.ces.2004.11.043

[7] Yu, Z., Cope, R.F., Kar, K.K., Evan, R.C. and Guillaudeu, S.J. (2012) Mixing Performance of the Novel Kar Dynamic Mixer Impeller in Small Laboratory-Scale Systems. Industrial \& Engineering Chemistry Research, 51, 15282-15292. https://doi.org/10.1021/ie3018548

[8] Ng, K.C. and Ng, E.Y.K. (2013) Laminar Mixing Performances of Baffling, Shaft Eccentricity and Unsteady Mixing in a Cylindrical Vessel. Chemical Engineering Science, 104, 960-974. https://doi.org/10.1016/j.ces.2013.10.031

[9] Matos, J., Brito, M.S.C.A., Dias, M.M., Lopes, J.C.B. and Santos, R.J. (2018) Lagrangian Mixing Simulation and Quantification of Scales. Chemical Engineering Science, 192, 199-210. https://doi.org/10.1016/j.ces.2018.07.035

[10] Goto, S., Shimizu, M. and Kawahara, G. (2014) Turbulent Mixing in a Precessing Sphere. Physics of Fluids, 26, Article ID: 115106.

[11] Zughbi, H.D. and Rakib, M.A. (2004) Mixing in a Fluid Jet Agitated Tank: Effects of Jet Angle and Elevation and Number of Jets. Chemical Engineering Science, 59, 829-842. https://doi.org/10.1016/j.ces.2003.09.044

[12] Cheng, D., Feng, X., Cheng, J. and Yang, C. (2013) Numerical Simulation of Macro-Mixing in Liquid-Liquid Stirred Tanks. Chemical Engineering Science, 101, 272-282. https://doi.org/10.1016/j.ces.2013.06.026

[13] Mishra, P. and Ein-Mozaffari, F. (2017) Using Computational Fluid Dynamics to Analyze the Performance of the Maxblend Impeller in Solid-Liquid Mixing Operations. International Journal of Multiphase Flow, 91, 194-207. https://doi.org/10.1016/j.ijmultiphaseflow.2017.01.009

[14] Lagrange, R., Meunier, P., Nadal, F. and Eloy, C. (2011) Precessional Instability of a Fluid Cylinder. Journal of Fluid Mechanics, 666, 104-145. https://doi.org/10.1017/S0022112010004040

[15] Chergui, N., Lateb, M., Lacroix, E. and Dufresne, L. (2015) CFD Study of Flow Dynamics in a Blade Free Planetary Mixer (BFPM) - A Qualitative Flow Study. Chemical Engineering Research and Design, 102, 100-115. https://doi.org/10.1016/j.cherd.2015.05.045

[16] Yamagata, T., Matsuzawa, T. and Fujisawa, N. (2017) Numerical Simulation of Flow Structure in Planetary Centrifugal Mixer. Proceedings of the Fourteenth Interna- 
tional Conference on Flow Dynamics, Sendai, 1-3 November 2017, 444-445.

[17] Danckwerts, P.V. (1952) The Definition and Measurement of Some Characteristics of Mixtures. Applied Scientific Research, Section A, 3, 279-296.

https://doi.org/10.1007/BF03184936

\section{Nomenclature}

\begin{tabular}{|c|c|}
\hline$d$ & vessel diameter $[\mathrm{mm}]$ \\
\hline$h$ & vessel height $[\mathrm{mm}]$ \\
\hline$I_{\mathrm{m}}$ & mixing index \\
\hline$N$ & number of sub-domains \\
\hline$n_{\text {rev }}$ & revolution speed $[\mathrm{rpm}]$ \\
\hline$n_{\text {rot }}$ & rotation speed $[\mathrm{rpm}]$ \\
\hline$p$ & pressure $[\mathrm{Pa}]$ \\
\hline $\operatorname{Re}$ & Reynolds number \\
\hline$R_{\mathrm{rev}}$ & revolution radius $[\mathrm{mm}]$ \\
\hline$r$ & position vector from a revolution axis \\
\hline$t$ & time $[s]$ \\
\hline$U_{0}$ & wall velocity $[\mathrm{m} / \mathrm{s}]$ \\
\hline$u$ & velocity vector $[\mathrm{m} / \mathrm{s}]$ \\
\hline$u, V, w$ & velocity components $[\mathrm{m} / \mathrm{s}]$ \\
\hline$x, y, z$ & Cartesian coordinates \\
\hline$\Gamma$ & precession rate $\left(=n_{\text {rev }} / n_{\text {rot }}\right)$ \\
\hline$v$ & kinematic viscosity $\left[\mathrm{m}^{2} / \mathrm{s}\right]$ \\
\hline$\rho$ & fluid density $\left[\mathrm{kg} / \mathrm{m}^{3}\right]$ \\
\hline$\rho_{i}$ & particle number density in the $i$ th sub-domain \\
\hline$\rho_{\mathrm{m}}$ & averaged number density \\
\hline$\sigma$ & standard deviation of number density \\
\hline$\Omega$ & revolution rate $[\mathrm{rad} / \mathrm{s}]$ \\
\hline$\omega_{\text {rot }}$ & rotation rate $[\mathrm{rad} / \mathrm{s}]$ \\
\hline
\end{tabular}

